\title{
ARTICLE
}

\section{Electron-induced fragmentation mechanisms in organic monomers and their implications on photoresist optimization for EUV Lithography}

Received 00th January 20xx, Accepted 00th January 20xx DOI: $10.1039 / x 0 \times x 00000 x$

\begin{abstract}
Ashish Rathore, ${ }^{* a, b}$ Maicol Cipriani, ${ }^{c}$ Ching-Chung Huang, ${ }^{d}$ Lionel Amiaud, ${ }^{e}$ Céline Dablemont, ${ }^{e}$ Anne Lafosse, ${ }^{\mathrm{e}}$ Oddur Ingólfsson, ${ }^{\mathrm{c}}$ Danilo De Simone, ${ }^{\mathrm{b}}$ Stefan De Gendta ${ }^{\mathrm{a}, \mathrm{b}}$

Secondary electrons generated during the Extreme Ultraviolet Lithography (EUVL) process are predominantly responsible for inducing important patterning chemistry in the photoresist film. Therefore, it is crucial to understand the electroninduced fragmentation mechanisms involved in the EUV-resist systems to improve their patterning performance. To facilitate this understanding, mechanistic studies were carried out on simple organic EUV-resist monomers, Methyl Isobutyrate (MIB), and Methacrylic Acid (MAA) both in condensed and gas phase. Electron-stimulated desorption (ESD) studies on MIB in the condensed phase showed peaks in desorption at around 2 and $9 \mathrm{eV}$ electron energies. The gas-phase study on MIB showed that the monomer followed the dissociative lonization (DI) fragmentation pathway, under single collision conditions, which opened up at electrons energies above about $11 \mathrm{eV}$. No signs of dissociative electron attachment (DEA) pathway were detected for MIB in the gas phase under single collision conditions. However, DEA was an active process in MAA in the gas phase under single collision conditions at around $2 \mathrm{eV}$, showing that slight modifications of the molecular structures of photoresists may serve to sensitize them to certain electron-induced processes.
\end{abstract}

\section{Introduction}

For the past few decades, Deep Ultraviolet Lithography (DUVL) has been used for the high-volume manufacturing (HVM) process by the semiconductor industry. DUVL process uses light with a wavelength of $193 \mathrm{~nm}$ (energy $\sim 6 \mathrm{eV}$ ) to print at a resolution down to $40 \mathrm{~nm}$ with a single patterning step. One of the major factors responsible for the success of the DUVL process was the application of the photoresist platform called Chemically Amplified Resists (CAR). CAR contains multiple components such as a base polymer chain, photoacid generators (PAG), quencher, other additives, and works efficiently (with high sensitivity) for DUVL.

For further miniaturization of microchips, Extreme Ultraviolet Lithography (EUVL) has recently been introduced as the new high-volume manufacturing (HVM) technology in the semiconductor industry. EUVL uses the light of wavelength $13.5 \mathrm{~nm}$ (energy $92 \mathrm{eV}$ ) to be able to print at sub-10 nm

\footnotetext{
a. KU Leuven Department of Chemistry, Celestijnenlaan 200F, 3001 Leuven, Belgium E-mail: ashish.rathore@imec.be

b. IMEC, Kapeldreef 75, 3001 Leuven, Belgium

c. Science Institute and Department of Chemistry, University of Iceland, Dunhagi 3, 107 Reykjavik, Iceland.

d. Institute of Materials Science and Technology, TU Wien, 1060 Wien, Austria

e. Université Paris-Saclay, CNRS, Institut des Sciences Moléculaires d'Orsay, 91405 Orsay, France.
}

Electronic Supplementary Information (ESI) available: [details of any supplementary information available should be included here]. See DOI: $10.1039 / x 0 x x 00000 x$ resolutions. But one of the major roadblocks prolonging the shift from DUVL to EUVL in the industry is the unavailability of a photoresist system that can work well with the new process.

Transferring the conventional CAR platform to the new process has been proving problematic due to issues related to resolution limitations, stochastic failure, and its complex blended chemistry. ${ }^{1-3}$ Therefore, alternative non-CAR systems are currently being developed and tested for EUVL, but need major optimizations before they can be applied to ultrahighresolution patterning. ${ }^{4}$ For example, non-CAR systems require a high EUV-dose to print dense features with acceptable roughness, which is non-ideal for HVM. Hence, it is important to improve the sensitivity of the resists, and the primary focus of the photoresist manufacturers to achieve this has been to incorporate metals with high EUV-absorbance in their new systems. ${ }^{5-8}$ However, this approach does not provide the desired results, as the fundamental understanding of the patterning mechanism of these alternative resist systems is incomplete. ${ }^{8}$

In our previous study to understand the EUVL-mechanism, ${ }^{9}$ we reported that the secondary electrons generated in the film are responsible for inducing most of the litho-chemistry in the photoresist. This indicates that it is crucial to understand the electron-induced mechanisms of the alternative non-CA EUV-resist systems, to optimize them for HVM.

In the conventional DUVL process with low photon energy, direct molecular excitation is responsible for activating the photoacid generator (PAG) molecules. ${ }^{10,11}$ However, when a photoresist film is exposed to EUV-photons of $92 \mathrm{eV}$, cascades 
of electrons of about $80 \mathrm{eV}$ and below are generated.9,12 These electrons can cause additional fragmentation reactions in the photoresist molecules through four distinct processes. ${ }^{13-15}$

(1) Dissociative Ionization (DI):

$$
\mathrm{AB}+\mathrm{e}^{-} \rightarrow \mathrm{AB}^{\#+}+2 \mathrm{e}^{-} \rightarrow \mathrm{A}^{\#+}+\mathrm{B}^{\#}+2 \mathrm{e}^{-}
$$

This is a direct, ionization event, in which energy transfer from an incident electron, with energy equal to or greater than the ionization threshold of the respective molecule, effectuates removal of a bound electron. This process is shown above for the diatomic $A B$, where the first step is the formation of the diatomic parent cation, $\mathrm{AB}^{\#+}$. Here the asterisk signifies that the cation formed may be vibrationally or electronically excited. If $\mathrm{AB}^{\#+}$ is above the threshold for dissociation, it may further fragment to form a neutral and a positive ion fragment. In the case of a polyatomic molecule, one or more neutral fragments may be formed. This is a non-resonant process, generally, with an onset slightly above the ionization energy of the respective molecule, a maximum in the range between 50 and $100 \mathrm{eV}$, and a gradual decline above that energy as the energy transfer efficiency decreases.

(2) Dissociative Electron Attachment (DEA):

$\mathrm{AB}+\mathrm{e}^{-} \rightarrow \mathrm{AB}^{\#-} \rightarrow \mathrm{A}^{\#-}+\mathrm{B}^{\#}$

This is a resonant process, during which an electron is captured by the parent molecule $A B$, forming a transient negative ion $\left(\mathrm{AB}^{\#-}\right)$. The transient negative ion then relaxes either through re-emission of the electron (auto-detachment, $A D$ ) or by fragmenting into an anion and one or more neutral fragments (DEA). The electron capture proceeds within narrow energy ranges, generally below the ionization limit of the respective molecules, and is most efficient at very low energies, close to the $0 \mathrm{eV}$ threshold.

(3) Neutral Dissociation (ND), and (4) Dipolar Dissociation (DD):

$$
\begin{aligned}
& \mathrm{AB}+\mathrm{e}^{-}\left(\epsilon_{1}\right) \rightarrow[\mathrm{AB}]^{*}+\mathrm{e}^{-}\left(\epsilon_{2}<\epsilon_{1}\right) \rightarrow \mathrm{A}^{\#}+\mathrm{B}^{\#} \\
& \mathrm{AB}+\mathrm{e}^{-}\left(\epsilon_{1}\right) \rightarrow[\mathrm{AB}]^{*}+\mathrm{e}^{-}\left(\epsilon_{2}<\epsilon_{1}\right) \rightarrow \mathrm{A}^{\#+}+\mathrm{B}^{\#-}
\end{aligned}
$$

These processes are both initiated by an electronic excitation within the respective molecule, leading in the case of the diatomic $\mathrm{AB}$, to an electronically excited state $[\mathrm{AB}]^{*}$. These are non-resonant processes with an onset at the first electronic excitations over the dissociation limit of the respective molecules. In the former case, the neutral electronic excited state $[A B]^{*}$ dissociates to form neutral fragments $\left(A^{\#}\right.$ and $\left.B^{\#}\right)$ in the latter ionic pairs $\left(A^{\#+}\right.$ and $\left.B^{\#-}\right)$. For polyatomic molecules, more than one neutral fragment may be formed. ${ }^{13-20}$
DI, DEA and, DD processes can be studied through Mass Spectrometry (MS), as they produce charged fragments. The neutral species may also be studied by mass spectrometry after desorption through post ionization e.g. using an electron bombardment ionization stage.

In this work, the electron-induced mechanism of an EUVresist monomer used in the non-CA resist, methyl isobutyrate $\left(\mathrm{C}_{5} \mathrm{H}_{10} \mathrm{O}_{2}\right.$, further referred to as MIB, structure presented in figure 1a), is studied in the film and gas-phase. Temperature and electron-stimulated desorption in ultrathin films of MIB are analyzed by a residual gas analyzer (RGA) set up. To complement the film-phase experiments and get information about some of the desorbed transient species that might remain in the film and cannot be picked up by the RGA, a gasphase study is also conducted in a crossed electron-molecule beam apparatus. To complete the mechanistic study, another monomer, methacrylic acid $\left(\mathrm{C}_{4} \mathrm{H}_{6} \mathrm{O}_{2}\right.$, further referred to as MAA, structure presented in figure $1 \mathrm{~b}$ ), is tested and results are compared with MIB. Finally, the significance of these results in improving the current EUV-photoresist systems to achieve better patterning performances is discussed. (a)

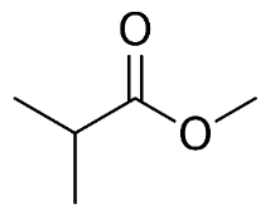

(b)

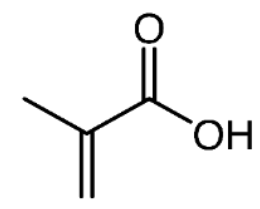

Fig. 1 Structures of two alternative non-CA-based EUV-resist monomers (a) Methyl Isobutyrate (MIB) and (b) Methacrylic Acid (MAA) used in this study.

\section{Experimental details}

\subsection{Materials}

MIB monomer (99\%), MAA monomer (99\%), and other reagents used in this work are obtained from Sigma-Aldrich.

\subsection{Methodology}

2.2.1 Temperature-programmed desorption (TPD) and electron-stimulated desorption (ESD) in film-phase. Temperature and electron-induced fragmentations in the resist film cause desorption of charged and neutral volatile species, which can be analyzed with a mass spectrometer with an ionization stage (RGA)

TPD and ESD analyses of MIB in the film-phase were carried out with the setup installed at the University of ParisSaclay for studying the electron-induced processes in thin molecular films. The tool was primarily an ultrahigh vacuum (UHV) chamber installed with the following components - a conductive $(\mathrm{Cu})$ sample holder attached to a cryostat, a doser equipment to deposit the sample onto the sample holder, an electron gun to expose the samples to low energy electrons (LEEs) and the RGA to analyze the desorbed species. Complete information on the tool can be found elsewhere. ${ }^{16,21,22}$ The 
typical background pressure in the UHV chamber was maintained at around $5 \times 10^{-10} \mathrm{mbar}$ with the sample introduced at a gas pressure in the range of (2-7) $\times 10^{-9} \mathrm{mbar}$.

To perform the analysis, a background pressure of MIB was exposed to a 70-eV electron beam, and a mass spectrum was recorded with the Quadruple Mass Spectrometer (QMS). The molecular fragmentation panel gave insights into potentially interesting fragments. Next, TPD experiments were carried out by depositing different thicknesses of MIB films onto the sample holder (by varying the dosing time) and heating them from 30-300K. Temperature-induced desorption species were analyzed with the RGA and an intensity vs temperature graph (known as the TPD curve) was obtained. The thickness of the initially deposited film was deduced from the shape of the TPD curve, and the deposition time was calculated using equation 5. The complete procedure for this is presented elsewhere ${ }^{23-25}$.

$$
\boldsymbol{t}_{\text {deposition }}=\frac{L}{\boldsymbol{P}_{\text {partial }(\text { monomer })}}
$$

Here, $t_{\text {deposition }}$ represents the deposition time, ' $\mathrm{L}$ ' refers to the Langmuir monolayer $\left(=10^{-6} \mathrm{mbar} . \mathrm{sec}\right)$, and $\mathrm{P}_{\text {partial(monomer) }}$ refers to the partial pressure of the monomer in the UHV chamber. This allowed the determination of the dosing time required to deposit 5-10 monolayers (MLs) of MIB onto the sample holder (see results and discussion below). Working within this range avoids the charging effect caused by thicker films. Electron stimulated desorption (ESD) experiments were carried out by exposing the ultrathin film of MIB to LEEs of $1-20 \mathrm{eV}$ and monitoring the energy dependence of the electron-induced desorption of individual species with the RGA as described in detail elsewhere. ${ }^{22}$ The steps to obtain electron-stimulated yields from the raw spectra are shown in figure S1 of the supplementary information.

2.2.2 Electrons-induced fragmentation in the gas-phase. Monomers in the gas-phase were exposed to an electron beam and the fragmentation mechanism was studied by MS

The gas-phase study of MIB was conducted in an electronmolecule crossed beam apparatus at the University of Iceland. It consisted of a Trochoidal Electron Monochromator (TEM), an effusive gas inlet system, and a QMS (Hiden EPIC 1000). The complete information on the tool can be found elsewhere ${ }^{26}$. A quasi-monoenergetic electron beam, generated with the trochoidal monochromator, crosses an effusive beam of the target molecules. The charged fragments resulting from the electron-molecule interaction were extracted by a small electric field (about $1 \mathrm{~V} / \mathrm{cm}$ ) and directed into the entrance aperture of the QMS to be $\mathrm{m} / \mathrm{z}$ analyzed and detected. For studies of DI processes, the instrument was run in positive ion mode, and for the studies of DEA in negative ion mode. Typical background pressure was around $4 \times 10^{-8} \mathrm{mbar}$, and the current experiments were carried out with a sample gas pressure in the range of (2-9) $\times 10^{-7} \mathrm{mbar}$.
Mass spectra of the positive ions formed were recorded at a fixed electron energy of $70-\mathrm{eV}$ by scanning through the relative mass range and ion yield curves were recorded at fixed mass by scanning over the relevant electron energy range.

To determine the appearance energies (AEs) of individual fragments the onset of the respective ion yields were fitted with a Wannier type function ${ }^{27}$ of the form:

$$
\begin{gathered}
\mathbf{E} \leq \mathbf{A E}, \mathbf{f}(\mathbf{x})=\mathbf{b} \\
\mathbf{E}>\mathbf{A E}, \mathbf{f}(\mathbf{x})=\mathbf{b}+\mathbf{a}(\mathbf{E}-\mathbf{A E})^{\mathbf{d}}
\end{gathered}
$$

Here, ' $E$ ' is the energy of the incident electron, ' $A E$ ' is appearance energy, ' $b$ ' is a constant related the to background signal, ' $a$ ' is a scaling coefficient, and ' $d$ ' is an exponential factor. In this process, the energy scale was calibrated using the well-established ionization energy of $\operatorname{argon}^{28}$ and three independent datasets, recorded on different days were fitted. The standard deviation of the mean was taken as the uncertainty for the respective AEs.

The DEA study of MAA was conducted in an electronmolecule crossed beam apparatus at the Siedlce University of Natural Sciences and Humanities. The energy scale was calibrated with the $\mathbf{S F}_{\mathbf{6}}^{-}$signal from $\mathbf{S F}_{\mathbf{6}}$ gas at $0 \mathrm{eV}$ and the molecules were introduced in the UHV chamber operating at $10^{-8}$ mbar. The electron energy (energy scan) of the fragments was measured in the range from approximately $0-12 \mathrm{eV}$ at each channel $(\mathrm{m} / \mathrm{z})$ of interest.

2.2.3 Quantum chemical calculations. Fragmentation mechanisms in the gas-phase were further studied by quantum chemical calculations of the thermochemical thresholds for the relevant processes. All calculations for the threshold energies were carried out using ORCA $^{29}$ version 4.2.1. Geometry optimization of the parent molecule and relevant fragments was carried out using density functional theory (DFT) at the B3LYP ${ }^{30-32} /$ def2-TZVP 33 level of theory with D3(BJ) dispersion correction ${ }^{34}$. Harmonic vibrational frequencies were also calculated at this level and they were used to derive zero-point vibrational energy, ZPVE, and thermal energy, $E_{\text {therm }}$, corrections. The vibrational frequencies confirmed that all structures were stationary points on the potential energy surface. Single-point energy calculations were then performed on the optimized geometries using the coupled-cluster method at the DLPNO-CCSD $(T)$ level of theory, ${ }^{35-37}$ using DZ/TZ extrapolation (def2-SVP30 and def2TZVP). The final threshold energy for a fragment $A^{+}$was obtained by equation (6).

$$
\mathrm{E}_{\text {th }}=\mathrm{E}(\mathrm{M}-\mathrm{A})+\mathrm{E}\left(\mathrm{A}^{+}\right)-\mathrm{E}(\mathrm{M})+\mathrm{ZPVE}+\mathrm{E}_{\text {therm }}
$$

$M$ refers to the parent molecule, and ( $M-A)$ represents the neutral species after the fragmentation of species $A$ from $M$.

\section{Results and discussion}

\section{$3.1 \mathrm{MIB}$}

Gas-phase fragmentation to identify important desorption masses: MIB gas molecules were introduced in the UHV 
chamber and analyzed by mass spectrometry at 70-eV ionization energy (figure 2), and the following important fragments were identified: m/z 102 (parent cation), m/z 87 (loss of $\mathrm{CH}_{3}$-group), $\mathrm{m} / \mathrm{z} 71$ (the loss of $\mathrm{CH}_{3} \mathrm{O}$ ), $\mathrm{m} / \mathrm{z} 59$ (loss of $\mathrm{CH}_{3} \mathrm{CHCH}_{3}$-group), $\mathrm{m} / \mathrm{z} 43\left(\mathrm{CH}_{3} \mathrm{CHCH}_{3}{ }^{+}\right)$, and $\mathrm{m} / \mathrm{z} 15$ (methyl cation). The structures of these fragments are provided in figure S2 of the supplementary information. The relative intensities of the $\mathrm{m} / \mathrm{z}$ ratios obtained here show good agreement with the spectrum available at the NIST database, ${ }^{38}$ as shown in the supplementary information figure $\mathrm{S} 3 . \mathrm{m} / \mathrm{z} 44$ $\left(\mathrm{CO}_{2}\right), \mathrm{m} / \mathrm{z} 28\left(\mathrm{CO} / \mathrm{N}_{2}\right)$, and $\mathrm{m} / \mathrm{z} 16, \mathrm{~m} / \mathrm{z} 17, \mathrm{~m} / \mathrm{z} 18(\mathrm{O}, \mathrm{OH}$, $\mathrm{H}_{2} \mathrm{O}$ ) along with low mass hydrocarbon fragments were mainly chamber contaminants.

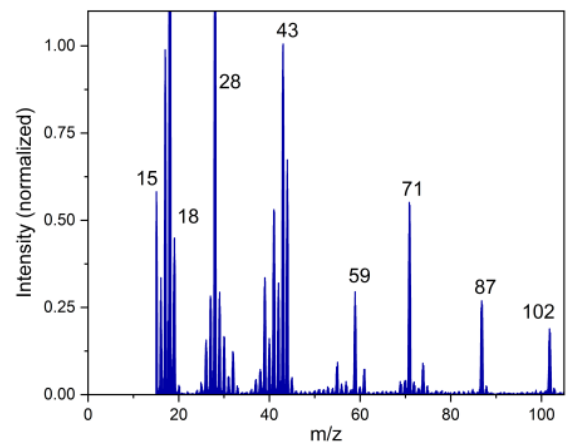

Fig. 2 70-eV electron-induced fragmentation mass spectrum of MIB introduced in the UHV setup.

The next step was to perform the TPD experiments to calculate the dosing time required to deposit 5-10 monolayers (ML) of MIB onto the sample holder to perform ESD experiments and avoid charging effects.

TPD analysis: A variable dosing time of 30,80 , and 240 seconds was used to deposit different thicknesses of MIB films onto the sample holder using the doser, and TPD analysis was carried out from $30-300 \mathrm{~K}$. Figure $3(\mathrm{a})$ presents the TPD curve of $\mathrm{m} / \mathrm{z}-43$, which shows that the desorption started at $120 \mathrm{~K}$ with the maximum at $145 \mathrm{~K}$. TPDs of other $\mathrm{m} / \mathrm{z}$ ratios are provided in figure $\mathrm{S} 4$ of the supplementary information. Figure 3 (b) suggests a linear deposition of molecules onto the sample holder with respect to the dosing time. Further, from the TPDcurve, it is seen that the deposition at 240s has two peaks. The first at $145 \mathrm{~K}$ is running high with increasing deposition time and is attributed to multilayer desorption. The second one, at $200 \mathrm{~K}$, would be then the desorption of the last monolayer (at the sample holder surface). The filling behavior of the profile from dose 30s, 80s, and 240s shows that the deposition is not uniform. Multilayering is starting to be visible at 30 seconds just after the first layer is completed. This is possible due to the very low deposition temperature (30K) and the high deposition rate of the doser. The comparison of the area of the second peak obtain with 240 seconds deposition and the full profile obtain with 30 seconds deposition time allows us to conclude that deposited quantities are $\sim 1 \mathrm{ML}, 4 \mathrm{ML}$, and 12 ML for doses 30 seconds, 80 seconds, and 240 seconds respectively. Therefore, a dosing time of 200 seconds is selected to deposit approx. $9 \mathrm{MLs}(1 \mathrm{~nm})$ and study the ESD of MIB monomers in the condensed phase.

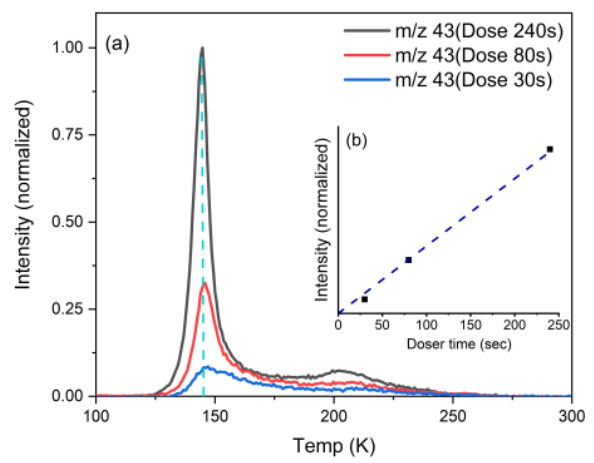

Fig. 3 (a) TPD curve of m/z-43; (b) Intensity (at 145K) vs dosing time plot shows a linear trend of deposition of MIB molecules onto the sample holder.

ESD analysis to check the electron energy dependence of fragment desorption from MIB ultrathin film: MIB ultrathin film $(1 \mathrm{~nm})$ was exposed to LEEs of $0-20 \mathrm{eV}$ at a temperature of $35 \mathrm{~K}$. The desorption yield of different neutral fragments was checked with the RGA and the result of $m / z 43$ is provided in figure 4 a. The desorption yield shows peaks at around 2 and 9 $\mathrm{eV}$, which suggests resonant fragment formation at these energies. Similar resonances were also observed in our previous study with $40 \mathrm{~nm}$ PMMA films (figure $4 \mathrm{~b}$ ). ${ }^{9}$ The peak intensity at $2 \mathrm{eV}$ (highlighted) was quite low for PMMA, which could be due to the inability of the fragments to diffuse through the film and reach the RGA setup. Further, the peak at $9 \mathrm{eV}$ for MIB matches the activation energy observed in the PMMA film, which refers to the minimum energy required to induce sufficient bond cleavages to produce volatile fragments that can be detected by the RGA. ${ }^{9}$

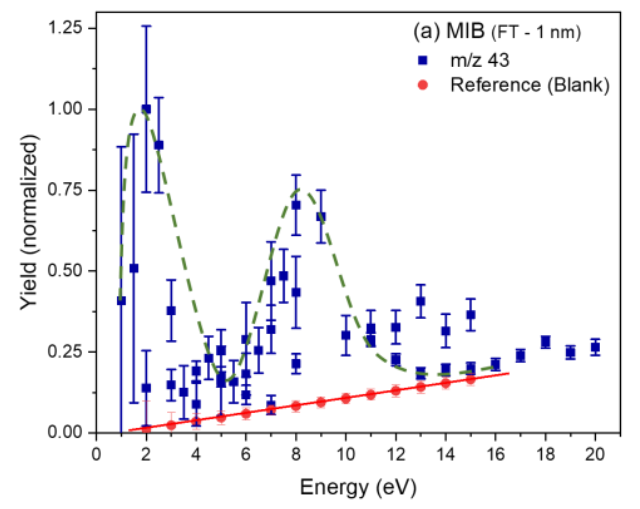

This journal is (C) The Royal Society of Chemistry 20xx 


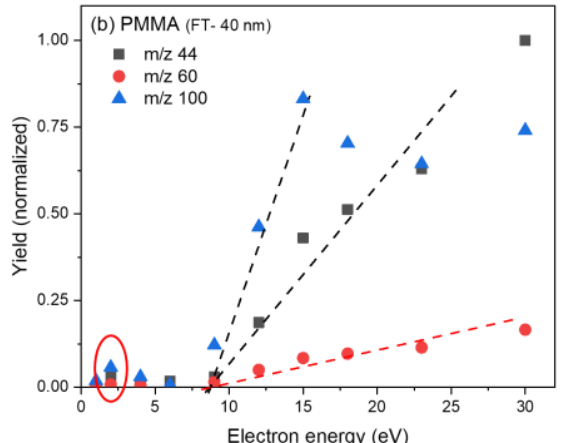

Fig. 4 (a) ESD curve of $\mathrm{m} / \mathrm{z}$ ratio 43 shows resonance fragmentation processes at approx. 2 and $9 \mathrm{eV}$ electron energies for MIB, same as, (b) peaks observed at 2 eV (highlighted in red) and $9 \mathrm{eV}$ (LEE-induced activation energy) for PMMA in our previous study (Adapted from [9]).

To try and shed some light on the apparent resonant behavior of the electron-induced desorption of the MIB film, a gas-phase study was conducted where MIB molecules interacted with low energy electrons of well-defined energy under single collision conditions and its fragmentation mechanisms were checked.

\section{The role of DI and DEA in MIB}

In this study, MIB gas molecules were exposed to LEEs to study the energy dependence of its fragmentation through $\mathrm{DI}$ and DEA.

DI: To attain a mass spectrum in the gas phase an effusive beam of MIB was crossed with an electron beam at 70-eV and the positive ions were analyzed by the QMS. The mass spectrum conforms well with that from NIST and that recorded with the RGA in the film phase experiment. The main fragments from MIB are of the $\mathrm{m} / \mathrm{z}$ ratios $102,87,74,71,59$, 43 , and 15 (figure $5 a$ ), and the $\mathrm{m} / \mathrm{z}$ ratios 18 and 28 are attributed to $\mathrm{N}_{2}$ and $\mathrm{H}_{2} \mathrm{O}$, respectively. The $\mathrm{m} / \mathrm{z}$ ratio 102 is attributed to the molecular ion and the $\mathrm{m} / \mathrm{z}$ ratios $87,74,71$, 59,43 , and 15 are all consistent with single bond ruptures. The $\mathrm{m} / \mathrm{z}$ ratio 74 , on the other hand, is attributed to CO loss and rearrangement to form the methyl, isopropyl ether. The AE of the molecular ion, i.e., the experimental value for the ionization energy of MIB, was found to be $10.1 \pm 0.4 \mathrm{eV}$ (figure $5 b), A E s$ for the $m / z$ ratio 59 and 43 were found to be $11.9 \pm$ $0.4 \mathrm{eV}$ (figure 5c) and $11.9 \pm 0.3 \mathrm{eV}$ (figure $5 \mathrm{~d}$ ), respectively, which were comparable to the values reported in the NIST database $^{38}$. For the ionization threshold $(\mathrm{m} / \mathrm{z}$ ratio 102$)$, a value of $9.4 \mathrm{eV}$ at the DFT level of theory was calculated, which was somewhat lower than the experimentally determined threshold. At the DLPNO-CCSD level of theory, on the other hand, the calculated threshold is $9.9 \mathrm{eV}$, which was in good agreement with the experimental value. For the $\mathrm{m} / \mathrm{z}$ ratio 43 , threshold values of 10.9 and $11.4 \mathrm{eV}$ were calculated at the DFT and DLPNO-CCSD level of theory, respectively. And for the complementary $\mathrm{m} / \mathrm{z}$ ratio 59 , the threshold values were calculated to be 11.0 and $11.4 \mathrm{eV}$ at the DFT and DLPNO-CCSD level of theory. Thus, it is clear that the rise in the $\mathrm{m} / \mathrm{z}$ ratio 43 desorption yield at about $6 \mathrm{eV}$, that peaks around $9 \mathrm{eV}$ cannot be due to $\mathrm{DI}$, which is not an open dissociation path below about $11 \mathrm{eV}$.
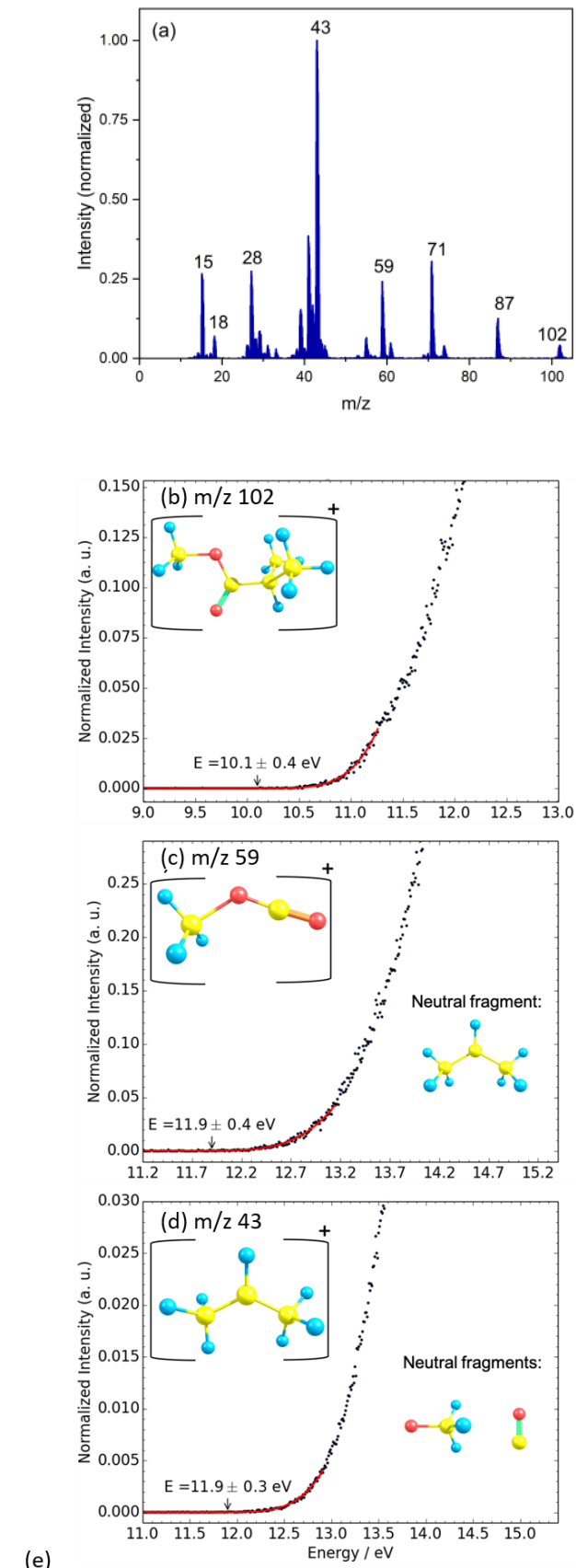

\begin{tabular}{ccc}
\hline Fragment & Experimental AE & $\begin{array}{c}\text { Theoretical } E_{\text {th }} \\
\text { DFT (DLPNO-CCSD) }\end{array}$ \\
\hline $\mathrm{m} / \mathrm{z} 102$ & $10.1 \pm 0.4 \mathrm{eV}$ & $9.4(9.9) \mathrm{eV}$ \\
$\mathrm{m} / \mathrm{z} 59$ & $11.9 \pm 0.4 \mathrm{eV}$ & $11.0(11.4) \mathrm{eV}$ \\
$\mathrm{m} / \mathrm{z} 43$ & $11.9 \pm 0.3 \mathrm{eV}$ & $10.9(11.4) \mathrm{eV}$ \\
\hline
\end{tabular}

Fig. 5 (a) Detection of several positive ions by QMS to confirm the presence of D mechanism in MIB molecules; (b) Experimental AE of the $\mathrm{m} / \mathrm{z} 102$ (parent cation) is determined to be $10.1 \pm 0.4 \mathrm{eV}$, (c) $\mathrm{m} / \mathrm{z} 59$ positive ion is determined to be $11.9 \pm 0.4 \mathrm{eV}$, and (d) $\mathrm{m} / \mathrm{z} 43$ positive ion is determined to be $11.9 \pm 0.3 \mathrm{eV}$. (e) The experimentally determined $\mathrm{AE}$ values are compared with the theoretically calculated values. 
DEA: Within the sensitivity of the instrument, no negative ions were detected under single collision conditions for the MIB molecules when these were exposed to LEEs in the energy range from about 0 to $10 \mathrm{eV}$. This shows that DEA is a negligible fragmentation process in LEE interaction with MIB under single collision conditions and indicates that DI and ND are rather the two major electron-induced fragmentation mechanisms in MIB molecules. However, as may be seen from figure 4a significant ESD desorption is observed at around 2 $\mathrm{eV}$. This is well below the first electronic excitation energies of MIB and the only possible electron-induced dissociation mechanism at such low electron energies is DEA. It is thus likely that this contribution is mediated through stabilization of the transient negative ion in the condensed phase allowing sufficient time for its relaxation through dissociation, while auto-detachment is the predominant relaxation mechanism under single collision conditions. Such stabilization has e.g., been observed for $\mathrm{Cl}$ - formation from $\mathrm{C}_{6} \mathrm{~F}_{5} \mathrm{Cl}$ and $\mathrm{CF}_{3}$ formation from $\mathrm{CF}_{3}$ l in clusters and condensed media. ${ }^{39}$

\subsection{MAA}

To further explore the possibility to stabilize such negative ion resonances by changing the molecular composition, additional DEA experiments were conducted on methacrylic acid (MAA) in the gas phase under single collision conditions. Here the methyl ester is replaced by the respective carboxylic acid and an additional double bond is introduced allowing for stabilization of the transient negative ion through conjugation. The main DEA channel observed for this compound is that of the formation of the dehydrogenated molecular anion, $\mathrm{CH}_{3} \mathrm{CCH}_{2} \mathrm{COO}^{-}$at $\mathrm{m} / \mathrm{z}$ ratio 85 . As is shown in figure 6 , the bulk of the $\mathrm{CH}_{3} \mathrm{CCH}_{2} \mathrm{COO}^{-}$ion yield from MAA is observed through a low-lying resonance reflected in a fairly narrow contribution at around $2 \mathrm{eV}$. This is similar to what has been observed in DEA to propionic acid, ${ }^{40}$ which in turn implies that the enhanced DEA efficiency observed for MMA as compared to MIB is due to the replacement of the methyl ester group with the carboxylic acid function, rather than the introduction of an additional double bond.

Nonetheless, this shows clearly that the molecular composition is critical and that by changing the molecular chemistry, it is indeed possible to open up the DEA fragmentation pathway.

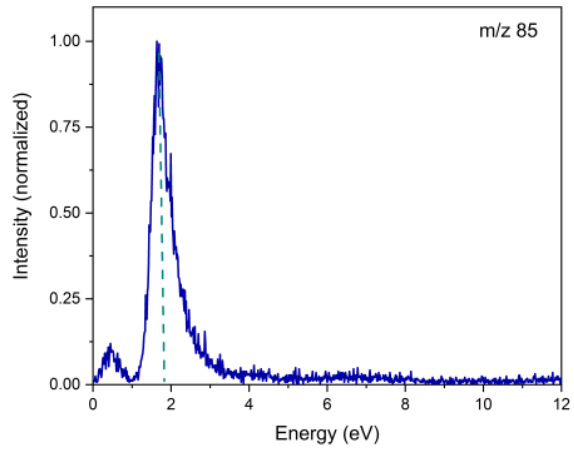

Fig. 6 DEA curve of amu- 85 of the MAA monomer shows resonance close to $2 \mathrm{eV}$.

This study has significance in designing better EUVphotoresists for the future. For example, for the past several years, photoresist manufacturers have tried to improve the EUV-sensitivity of their resist systems by simply incorporating metals with a high EUV-absorption. ${ }^{5-8}$ However, in many cases, this approach does not directly replicate to better EUVL performance. A better approach to improve the resist sensitivity is by opening up multiple electron-induced fragmentation pathways in the photoresist system. This can be done by adding appropriate functional groups that can use a wide array of LEEs generated in the film to open up multiple chemical pathways. Another noticeable point is that since the electron-induced fragmentation of a molecule is enhanced only at specific energies (as seen in figure 3a), it might be possible to modulate photoresist chemistry to open up specific reaction channels that can improve its patterning performance. ${ }^{41}$

\section{Conclusions}

This study was conducted to better understand the electroninduced mechanisms of the two EUV-resist monomers, Methyl Isobutyrate (MIB) and Methacrylic acid (MAA). First, the filmphase study of MIB involved temperature-programmed desorption (TPD) and electron-stimulated desorption (ESD) analyses, to understand the effect of temperature and electrons respectively. TPD analysis showed that the desorption of different masses starts at $125 \mathrm{~K}$ and the desorption peak maximum lies at 145K. ESD analysis of neutral fragments showed peak desorption at 2 and $9 \mathrm{eV}$. Next, gasphase studies were conducted to check for two major electron-induced fragmentation mechanisms, Dissociative Ionization (DI) and Dissociative Electron Attachment (DEA), in MIB molecules. The study indicated that ND and DEA are likely the two predominant mechanisms in the MIB molecule in the condensed phase. Whereas, no evidence of DEA was found under single collision conditions in the gas phase. Finally, the MAA monomer was tested to confirm that it is possible to open up the DEA pathway by molecular modification. This study has high significance on EUV-resist optimizations as it shows that by modifying the photoresist chemistry, it is possible to open-up new fragmentation pathways and induce specific chemical reactions to improve their EUVL-performance.

\section{Conflicts of interest}

There are no conflicts to declare.

\section{Acknowledgments}

MC acknowledges the doctoral grant from the University of Iceland Research Fund. $\mathrm{CCH}$ acknowledges Prof. Jürgen Stampfl (TU Wien) and Siedlce University of Natural Sciences 
and Humanities to facilitate this work. This project has received funding from the European Union's Horizon 2020 research and innovation program under the Marie Skłodowska-Curie grant agreement No 722149 with the acronym 'ELENA', which provides a PhD grant funding for AR.

\section{References}

1 P. P. Naulleau, C. N. Anderson, L. M. Baclea-An, P. Denham, S. George, K. A. Goldberg, G. Jones et al., Advances in Resist Materials and Processing Technology XXVIII SPIE, 2011, 7972, 797202.

2 S. Tarutani, H. Tamaoki, H. Tsubaki, T. Takahashi, H. Takizawa, H. Takahashi and S. J. Kang, Journal of Photopolymer Science and Technology, 2011, 24(2), 185-191.

3 L. Li, X. Liu, S. Pal, S. Wang, C. K. Ober and E. P. Giannelis, Chemical Society Reviews, 2017, 46(16), 4855-4866.

4 D. De Simone, V. Rutigliani, G. Lorusso, P. De Bisschop, Y. Vesters, V. B. Carballo and G. Vandenberghe, EUV Lithography IX SPIE, 2018, 10583, 105830G.

5 D. De Simone, Y. Vesters and G. Vandenberghe, Advanced Optical Technologies, 2017, 6(3-4), 163-172.

6 M. Murphy, A. Narasimhan, S. Grzeskowiak, J. Sitterly, P. Schuler, J. Richards, G. Denbeaux and R. L. Brainard, EUV Lithography VIII SPIE, 2017, 10143, 1014307.

7 P. De Bisschop and E. Hendrickx, Extreme Ultraviolet (UV) Lithography IX SPIE, 2018 10583, 105831K.

8 A. Robinson and R. Lawson, Materials and Processes for Next Generation Lithography, Elsevier, 2011, vol.11.

9 A. Rathore, I. Pollentier, H. Singh, R. Fallica, D. De Simone and S. De Gendt, Journal of Materials Chemistry C, 2020, 8(17), 5958-5966.

10 R. L. Brainard, C. Henderson, J. Cobb, V. Rao, J. F. Mackevich, U. Okoroanyanwu, S. Gunn, J. Chambers and S. Connolly, Journal of Vacuum Science \& Technology B, Microelectronics and Nanometer Structures Processing, Measurement and Nanometer, 1999, 17(6), 3384-3389.

11 S. Enomoto and T. Kozawa, Journal of Vacuum Science \& Technology B, Nanotechnology and Microelectronics: Materials, Processing, Measurements and Phenomena, 2018, 36(3), 031601.

12 J. W. Thackeray, Journal of Micro/Nanolithography, MEMS and MOEMS, 2011, 10(3), 033009.

13 Ingólfsson, O. Low energy electrons: Fundamentals and Applications. CRC Press 2019.

14 R. M. Thorman, T. P. R. Kumar, D. H. Fairbrother and $\mathrm{O}$. Ingólfsson, Beilstein Journal of nanotechnology, 2015, 6(1), 1904-1926.

15 M. Cipriani, R. M. Thorman, C. R. Brewer, L. McElwee-White and O. Ingólfsson, The European Physical Journal D, 2019 73(10), 227.

16 L. A. Sala, Low-Energy Electron Induced Chemistry in Supported Molecular Films, PhD thesis, University of ParisSaclay, 2018.

17 J. Schaefer and J. Hoelzl, Thin Solid Films, 1972, 13(1), 81-86.

18 A. Lafosse, M. Bertin and R. Azria, Progress in surface science, 2009, 84(5-6), 177-198.

19 L. Amiaud, J. Houplin, M. Bourdier, V. Humblot, R. Azria C. M. Pradier and A. Lafosse, Physical Chemistry Chemical Physics, 2014, 16(3), 1050-1059.

20 J. Houplin, C. Dablemont, L. Sala, A. Lafosse and L. Amiaud, Langmuir, 2015, 31(50), 13528-13534.

21 J. Houplin, L. Amiaud, V. Humblot, I. Martin, E. Matar, R. Azria, Physical Chemistry Chemical Physics, 2013, 15(19), 7220-7227.
22 L. Sala, I. B. Szymańska, C. Dablemont, A. Lafosse and L. Amiaud, Beilstein journal of nanotechnology, 2018, 9(1), 57 65.

23 H. Schlichting and D. Menzel, Surface science, 1992, 272(1-3) 27-33.

24 S. Conti, Computational studies of molecular self-assembly at surfaces: from rational design to function, PhD thesis, 2016.

25 C. Bronner and P. Tegeder, New Journal of Physics, 2014 16(5), 053004.

26 E. H. Bjarnason, B. Ómarsson, S. Engmann, F. H. Ómarsson and O. Ingólfsson, The European Physical Journal D, 2014, 68(5), 121.

27 G. H. Wannier, Physical Review, 1953, 90(5), 817.

28 R. C. Wetzel, F. A. Baiocchi, T. R. Hayes and R. S. Freund, Physical Review A, 1987, 35(2), 559.

29 F. Neese, Wiley Interdisciplinary Reviews: Computational Molecular Science, 2012, 2(1), 73-78.

30 A. D. Becke, The Journal of Chemical Physics, 1993, 98(7), 5648-5652.

31 C. Lee, W. Yang and R. G. Parr, Physical Review B, 1998 37(2), 785.

32 P. J. Stephens, F. J. Devlin, C. F. Chabalowski and M. J. Frisch, 1994, 98(45), 11623-11627.

33 F. Weigend and R. Ahlrichs, Physical Chemistry Chemical Physics, 2005, 7(18), 3297-3305.

34 S. Grimme, J. Antony, S. Ehrlich and H. Krieg, The Journal of chemical physics, 2010, 132(15), 154104.

35 C. Riplinger and F. Neese, The Journal of chemical physics, 2013, 138(3), 034106.

36 C. Riplinger, B. Sandhoefer, A. Hansen, F. Neese, The Journal of Chemical Physics, 2013, 139(13), 134101.

37 C. Riplinger, P. Pinski, U. Becker, E. F. Valeev and F. Neese, The Journal of Chemical Physics, 2016, 114(2), 024109.

38 NIST database [https://webbook.nist.gov/chemistry/].

39 O. Ingólfsson, F. Weik and E. Illenberger, International Journal of Mass Spectrometry and Ion Processes, 1996, 155 1-68.

40 A. Pelc, W. Sailer, P. Scheier, T. D. Märk and E. Illenberger Chemical Physics Letter, 2004, 392(4-6), 465-469.

41 E. Illenberger, H. U. Scheunemann and $H$. Baumgärtel, Chemical Physics, 1979, 37(1), 21-31. 